Pesq. Vet. Bras. 37(6):630-636, junho 2017

DOI: $10.1590 / \mathrm{S} 0100-736 \mathrm{X} 2017000600017$

\title{
Bem-estar dos cordeiros submetidos ao transporte rodoviário e avaliação das carcaças e carnes ${ }^{1}$
}

\author{
Fredson V. Silva ${ }^{2,3 *}$, Iran Borges ${ }^{3}$, Ângela M.Q. Lana ${ }^{3}$, Ana L.C.C. Borges ${ }^{3}$, Hemilly \\ C.M. Sá ${ }^{3}$, Vandenberg L. Silva ${ }^{3}$, Leonardo R.N. Alves ${ }^{3}$ e Fernando A. Souza ${ }^{3}$
}

\begin{abstract}
Silva F.V., Borges I., Lana A.M.Q., Borges A.L.C.C., Sá H.C.M., Silva V.L., Alves L.R.N. \& Souza F.A. 2017. [Welfare of lambs subjected to road transport and assessment of carcasses and meat.] Bem-estar dos cordeiros submetidos ao transporte rodoviário e avaliação das carcaças e carnes. Pesquisa Veterinária Brasileira 37(6):630-636. Departamento de Ciências Agrárias, Universidade Estadual de Montes Claros, Av. Reinaldo Viana s/n, Janaúba, MG 39440-000, Brazil. E-mail: fredson.silva@unimontes.br

This study aimed to assess the level of welfare in lambs by road transport and their carcasses and meat. Thus, we assessed behavioral parameters during transport, physiological parameters after landing and before slaughter and carcass and meat characteristics of lambs. Four road transports were achieved with increasing durations $(1 \mathrm{~h} 45 \mathrm{~min}, 3 \mathrm{~h} 52 \mathrm{~min}$, $7 \mathrm{~h} 30 \mathrm{~min}$ and $10 \mathrm{~h} 30 \mathrm{~min}$ ), there were twenty lambs in each transport. Animals body weight was $36.64 \pm 2.13 \mathrm{~kg}$ before transport. The lambs were slaughtered 15 hours after landing. The lambs lie down (median equal to zero every $20 \mathrm{~min}$ ) for a short time in journeys shorter than $3 \mathrm{~h} 52 \mathrm{~min}$. The number of potentially traumatic events is low (median near zero every 20min) for any transport duration. The adrenaline and cortisol concentrations, as well as metabolites that are controlled by them, did not testify that longer transport. However, the carcasses mass decreased and creatine kinase concentrations increased linearly with longer transports, which may reveal decrease in welfare. The meat quality of lambs was not influenced by the transport duration.
\end{abstract}

INDEX TERMS: Welfare, lambs, transport, carcasses, meat, stress, pre-slaughter, sheep.

RESUMO.- Objetivou-se avaliar o grau de bem-estar dos cordeiros submetidos ao transporte rodoviário e suas carcaças e carnes. Para isto, fez-se a avaliação dos parâmetros comportamentais durante o transporte, dos parâmetros fisiológicos após o desembarque e antes do abate e a caracterização das carcaças e carnes dos cordeiros. Realizaram-se quatro transportes rodoviários com durações crescentes (1h45min, 3h52min, 7h30min e 10h30min), cada transporte continha vinte cordeiros. 0 peso corporal dos animais foi de $36,64 \pm 2,13 \mathrm{~kg}$ antes do transporte. Os cordeiros foram abati-

\footnotetext{
${ }^{1}$ Recebido em 17 de junho de 2016.

Aceito para publicação em 18 de outubro de 2016.

${ }^{2}$ Departamento de Ciências Agrárias, Universidade Estadual de Montes Claros (Unimontes), Av. Reinaldo Viana s/n, Bico da Pedra, Janaúba, MG 39440-000, Brasil. *Autor para correspondência: fredson.silva@ unimontes.br

${ }^{3}$ Departamento de Zootecnia, Escola de Veterinária, Universidade Federal de Minas Gerais (UFMG), Av. Antônio Carlos 6627, Cx. Postal 567, Belo Horizonte, MG 30123-970, Brasil. E-mails: iran@vet.ufmg.br, lana@ vet.ufmg.br, analuiza@vet.ufmg.br, hemilly.mg@hotmail.com, berglira@ gmail.com, leo.rago@hotmail.com, nandodesouza@gmail.com
}

dos 15 horas após o desembarque. Os cordeiros deitaram por pouco tempo (mediana igual à zero a cada $20 \mathrm{~min}$ ) em jornadas menores que $3 \mathrm{~h} 52 \mathrm{~min}$. 0 número de eventos potencialmente traumáticos foi baixo (mediana próxima a zero, a cada $20 \mathrm{~min}$ ) para quaisquer durações dos transportes. As concentrações de adrenalina e cortisol, bem como os metabólitos que são controlados por eles, foram semelhantes entre os tratamentos. Contudo, a massa das carcaças diminuiu e as concentrações de creatina quinase aumentaram linearmente quando os transportes foram mais longos, o que podem revelar diminuição do bem-estar. A qualidade da carne de cordeiros não sofreu interferências da duração dos transportes.

TERMOS PARA INDEXAÇÃO: Bem-estar, cordeiros, transporte rodoviário, carcaças, carnes, estresse, pré-abate, ovinos.

\section{INTRODUÇÃO}

No período em que antecede o abate, várias são as etapas que os animais domésticos não são familiarizados (Broom \& Fraser 2010), dentre elas, o transporte. Este evento pode afetar o bem-estar dos animais, contudo, as condições que 
são realizadas podem determinar o grau do estresse (Tarrant 1990, Fisher et al. 2010). A perda de peso corporal, de acordo com a duração das jornadas, e seu efeito sobre as carcaças e carnes, são motivos de preocupação para a indústria e são constantemente investigados (Carter \& Gallo 2008, Tadich et al. 2009, Leme et al. 2012).

Como o estresse é uma avaliação complexa, deve-se rodeá-la de variáveis para se ter acurácia na compreensão do evento (Moberg \& Mench 2000). Variações relativas ao comportamento, às respostas dos sistemas nervoso autônomo, endocrinológico e imunológico são constantemente avaliadas com este objetivo (Hales \& Brown 1974, Ferguson \& Warner 2008, Cockram et al. 2010, Fisher et al. 2010).

Além dos problemas referentes ao bem-estar dos animais, ponto que não deve ser negligenciado por questões éticas, o aumento do estresse pode interferir nas características das carcaças e carnes (Gomide et al. 2006). As perdas referentes à capacidade de retenção de água das carnes, por exemplo, custam milhões de dólares a indústria (Huff-Lonergan \& Lonergan 2005), além de interferir em atributos como a maciez e a coloração (Kadim et al. 2006).

No Brasil não existem recomendações específicas nesta vertente para ovinos, portanto, fazem-se necessárias, já que a diferença dos genótipos aliada às condições edafoclimáticas distintas podem gerar resultados divergentes.

Diante do exposto, objetivou-se avaliar o grau de bem- estar de cordeiros submetidos ao transporte rodoviário e o impacto do estresse relacionado ao tempo de deslocamento em suas características de carne e carcaça.

\section{MATERIAL E MÉTODOS}

Todos os procedimentos experimentais foram aprovados pelo Comitê de Ética em Experimentação Animal (CETEA) da UFMG sob o número 185/2011.

Empregaram-se 80 cordeiros mestiços Santa Inês x Dorper, não-castrados com peso corporal de $36,64 \mathrm{~kg}$, desvio padrão da média $=2,13 \mathrm{~kg}$, no momento do transporte.

Os cordeiros ficaram em piquete coletivo que continha ração, de acordo com as recomendações do NRC (2007) para ganhos de $200 \mathrm{~g} \mathrm{dia}^{-1}$ para animais de $30 \mathrm{~kg}$, composta por fubá de milho, farelo de soja, bicarbonato de sódio, feno de Tifton 85 , suplemento mineral e água, sendo que o volumoso foi fornecido como $50 \%$ do total da matéria seca da dieta. Visando melhor uniformidade dos cordeiros, esses foram confinados por 30 dias até o transporte para o abatedouro frigorífico. Todos os cordeiros possuíam aproximadamente seis meses de idade, com peso médio de 36,64ะ2,13 kg.

Realizaram-se quatro transportes com vinte animais em cada um deles. As quilometragens percorridas foram 54, 199, 376 e 509 $\mathrm{km}$ e a duração foi de $1 \mathrm{~h} 45 \mathrm{~min}, 3 \mathrm{~h} 52 \mathrm{~min}, 7 \mathrm{~h} 30 \mathrm{~min}$ e $10 \mathrm{~h} 30 \mathrm{~min}$, respectivamente. 0 peso médio e desvio-padrão, em quilos, dos animais para cada um dos quatro transportes foi de $38,15 \pm 0,34$, $35,44 \pm 0,47,34,27 \pm 0,15$ e $34,25 \pm 0,27$. O espaço no caminhão foi $0,225 \pm 0,01 \mathrm{~m}^{2}$ animal $^{-1}$ de $36,64 \mathrm{~kg}$, como recomendado por Petherick \& Phillips (2009).

O veículo comercial (Volkswagen 8140, ano 1997) estava em bom estado de conservação e manutenção em ordem. A carroceria possuía duas caixas interligadas com um piso emborrachado, cada uma tinha dimensões de 5,00 x 2,14m, sendo que se utilizou a caixa próxima à cabine do motorista.

Vinte e seis horas antes do início do transporte, pesou-se os animais que já se encontravam em jejum de sólidos por doze ho- ras. Esta primeira pesagem foi utilizada para determinação das áreas disponíveis por animal. Após este procedimento, o arraçoamento foi normalizado. No dia do transporte, coletou-se o sangue dos cordeiros duas horas antes do embarque por punção da veia jugular, utilizando-se o sistema Vacutainer ${ }^{\circledR}$ (BD Diagnostics, São Paulo, Brasil). Os transportes deram-se na região central de Minas Gerais, Brasil, no entorno de Belo Horizonte. A principal rodovia trafegada foi a BR-040 no sentido de Belo Horizonte a Três Marias. $\mathrm{O}$ destino de todas as jornadas foi o município de Matozinhos. A velocidade do veículo não ultrapassou $65 \mathrm{~km} \mathrm{~h}^{-1}$. Os transportes foram realizados pelo mesmo motorista.

0 embarque dos cordeiros procedeu-se seguindo normas técnicas que zelam pelo bem-estar dos animais (Paranhos Da Costa et al. 2013), por meio de imobilização pelas regiões do abdômen e esterno.

Todo o percurso dos transportes foi filmado utilizando-se Kit DVR Encore ${ }^{\circledR}$ Enxdvr-4c Vigilância Digital. 0 aparelho possuía quatro câmeras com resolução de gravação de 640x272, conectadas a um gravador de vídeo digital e a um monitor de vídeo.

As medianas das temperaturas do ar $\left({ }^{\circ} \mathrm{C}\right)$ foram 29,30; 28,70; 31,90 e 27,90 para os transportes de $1 \mathrm{~h} 45 \mathrm{~min}, 3 \mathrm{~h} 52 \mathrm{~min}, 7 \mathrm{~h} 30 \mathrm{~min}$ e $10 \mathrm{~h} 30 \mathrm{~min}$, respectivamente. As medianas das umidades relativa do ar foram 48,$15 ; 41,60 ; 54,40$ e 70,45 para os transportes de $1 \mathrm{~h} 45 \mathrm{~min}, 3 \mathrm{~h} 52 \mathrm{~min}, 7 \mathrm{~h} 30 \mathrm{~min}$ e $10 \mathrm{~h} 30 \mathrm{~min}$, respectivamente. Estas medições foram realizadas por três dataloggers da marca Extech RHT10, programados para medições a cada 5 minutos. Os aparelhos foram colocados aleatoriamente no pescoço de três animais.

0 registro do comportamento animal deu-se por meio da análise visual dos vídeos. Estes registros foram feitos em um etograma previamente elaborado. Os eventos (ruminar, desequilibrar, cair, lutar, cabeçada, puxar a lã de outros animais) foram pontuais e o estado (em pé ou deitado) foi monitorado em intervalos. Todos os registros foram apresentados em intervalos de vinte minutos. Segue abaixo detalhamento das variáveis (Quadro 1). 0 posicionamento da orelha foi apresentado descritivamente.

Devido ao baixo número de ocorrências de agressões (lutar, cabeçada, puxar a lã de outros animais), essas variáveis foram analisadas juntas. 0 mesmo critério foi utilizado para saltar e andar sobre outros animais.

Os cordeiros foram desembarcados às $17 \mathrm{~h}, 17 \mathrm{~h}, 17 \mathrm{~h} 10 \mathrm{~min}$ e 18h30min para os transportes com duração de 1h45min,

\section{Quadro 1. Descrição dos eventos e estado utilizados para avaliação do comportamento de cordeiros durante o transporte rodoviário}
1. Postura
Deitar
Ruminar
Saltar sobre
Decúbito lateral ou ventral
$\begin{array}{ll} & \text { com exceção de tentativa de cópula } \\ \text { Andar sobre } & \text { Mudança de localização voluntária sobre um }\end{array}$
Movimento da mandíbula característico ou mais animais
2. Perdas de estabilidade
Desequilibrar Pequenos movimentos dos pés com rápidos movimentos das cinturas pélvica e escapular
Cair Queda rápida do corpo, resultando em contato com o chão
3. Agressão
Lutar
Cabeçada
Puxar lã
5. Vocalização
Vocalizar
Cabeçadas entre dois ou mais animais Contato voluntário da cabeça deste animal em quaisquer partes do corpo de outro Puxar lã de outro animal
Abertura da boca que caracteriza a emissão de sons 
$3 \mathrm{~h} 52 \mathrm{~min}, 7 \mathrm{~h} 30 \mathrm{~min}$ e $10 \mathrm{~h} 30 \mathrm{~min}$, respectivamente. Imediatamente após o desembarque no abatedouro frigorífico, colheu-se sangue dos cordeiros. Os ovinos ficaram em local coberto, com piso cimentado e com acesso a água. Após quatorze horas do desembarque, colheu-se novamente sangue dos animais.

As variáveis cortisol, proteínas totais, albumina, creatina quinase (CK), glicose, lactato e ácidos graxos não esterificados (AGNE) foram determinadas antes do transporte, imediatamente após o transporte (desembarque) e antes do abate (pré-abate). A variável adrenalina foi medida antes e após o transporte (desembarque).

Para todas as análises, utilizou-se o método colorimétrico de acordo com as especificações dos manuais dos kits. Foi utilizado analisador automático Cobas Mira ${ }^{\circledR}$ Plus para medição no sangue das proteínas totais, albumina, CK, glicose, lactato e AGNE, cada qual com seu respectivo kit comercial. Para medições das proteínas totais, albumina, creatina quinase, glicose foram utilizados kits da marca Synermed ${ }^{\circledR}$. Para medições do lactato e AGNE, utilizaram-se kits Lactato K084 da Bioclin ${ }^{\circledR}$ e FA 115 da Randox ${ }^{\circledR}$, respectivamente. Adrenalina e cortisol foram determinados no leitor de ELISA (Enzyme-LinkedImmunosorbentAssay), cada um com seu respectivo kit comercial. Adrenalina foi determinada com kit da Imuno-BiologicalLaboratories (IBL-America ${ }^{\circledR}$ ) e o cortisol com kitThe EIAsy Way -Cortisol distribuído por USA Diagnóstica ${ }^{\circledR}$.

0 procedimento de abate começou após quinze horas de descanso. A insensibilização deu-se por meio do sistema de eletronarcose seguido de sangria imediata. $\mathrm{O}$ abate de cada animal foi realizado aleatoriamente em um abatedouro frigorífico com Serviço de Inspeção Estadual. Todos os procedimentos estavam de acordo com o Regulamento Inspeção Industrial e Sanitária de Produtos Animais (Brasil 1997).

Aos quarenta e cinco minutos após a sangria, mediu-se o $\mathrm{pH}$, com peagâmetro(Sentron ${ }^{\circledR} 1001 \mathrm{pH}$ Meter), no músculo longissimus na altura da $12^{\text {a }}$ costela. As carcaças quentes receberam nota de escore de gordura subcutânea de acordo com Sañudo et al. (1997).

As carcaças foram pesadas e levadas para câmara fria com temperatura em torno de $2^{\circ} \mathrm{C}$ por vinte e quatro horas. Após o procedimento de resfriamento, foram novamente pesadas para obtenção dos pesos das carcaças frias. Depois da recalibração do peagâmetro, o $\mathrm{pH}$ das carcaças foi novamente medido após o resfriamento. Com o peso das carcaças quente e fria, obteve-se o rendimento de carcaças quente e fria, respectivamente, para isto, utilizou-se o peso corporal em jejum obtido antes do transporte. No transporte de 3h52min foi perdido as mensurações de pH inicial e final.

Parte do músculo longissimus das carcaças, em torno de 10 $\mathrm{cm}$ na região da $12^{\mathrm{a}}$ costela, foram retiradas após vinte e quatro horas de resfriamento, embalados a vácuo e congelados a $-20^{\circ} \mathrm{C}$. Após um mês, avaliaram-se, então, as colorações do músculo, capacidade de retenção de água e força de cisalhamento.

Em cada corte, após o descongelamento, foram retiradas duas fatias com 2,54 cm para medições posteriores. Mediu-se a força de cisalhamento pelo método proposto por Wheeler et al. (2005).

Depois da extração das duas fatias ainda cruas, a parte que sobrou do longissimus foi utilizada para a avaliação da capacidade de retenção de água, conforme método descrito por Nakamura \& Katoh (1985).

As determinações da cor da carne foram realizadas com um espectrofotômetro Hunter, modelo Miniscan EZ no sistema CIE. Avaliou-se a luminosidade ( $\left.\mathrm{L}^{*}\right)$, os teores da cor vermelha (a*) e da cor amarela ( $b^{*}$ ). A calibração do aparelho foi realizada antes da leitura das amostras com um padrão branco e outro preto. A coloração da carne foi medida nos cortes descongelados no lado em que não foram expostos à queima direta pelo frio. As seções da carne ficaram expostas ao ambiente por 30 minutos antes da realização das avaliações, com quatro réplicas por amostra.

0 delineamento foi inteiramente ao acaso, sendo que o transporte foi a variável independente. Utilizou-se o peso corporal em jejum como covariável $(\mathrm{p}<0,05)$ para ajustes nas variáveis dependentes pesos de carcaças quente e fria, $\mathrm{pH}$ inicial e final, capacidade de retenção de água, força de cisalhamento e coloração. As concentrações de cortisol, adrenalina, glicose, lactato, CK, proteínas totais, albumina e AGNE, medidas antes do transporte, também foram covariáveis das suas respectivas medições depois do transporte. Para cada variável dependente, testou-se a normalidade, a homogeneidade e aditividade do modelo $(p \geq 0,05)$. Após a análise de variância e teste " $F$ " $(p<0,05)$, as variáveis foram submetidas à análise de regressão $(\mathrm{p}<0,05)$ nos dois momentos, após o desembarque e antes do abate. As variáveis comportamentais e o escore de cobertura de gordura foram analisados pelo Teste Kruskal-Wallis $(\mathrm{p}<0,05)$.

\section{RESULTADOS}

Os cordeiros deitaram por mais tempo a partir do transporte que durou 7h30min (Quadro 2).

A duração das ruminações foi curta (menos que $1 \mathrm{mi}-$ nuto) e a frequência foi baixa (mediana próxima de zero em 20min). Mesmo assim, cordeiros que foram transportados nas jornadas de $7 \mathrm{~h} 30 \mathrm{~min}$ e $10 \mathrm{~h} 30 \mathrm{~min}$ ruminaram mais vezes do que os que estavam nos transportes $1 \mathrm{~h} 45 \mathrm{~min}$ e $3 \mathrm{~h} 52 \mathrm{~min}$. Os animais ficaram durante todo o transporte com as orelhas armadas e pescoço em posição vertical. Sete dos oitenta animais vocalizaram e, com exceção de dois cordeiros, apenas uma vez ( $p>0,05)$.

Os comportamentos potencialmente traumáticos apresentados durante os transportes estão no Quadro 3. Todas as ocorrências destas variáveis foram baixas (mediana próxima de zero em $20 \mathrm{~min}$ ) em quaisquer tratamentos. 0 somatório destas ocorrências também foi baixo (menor que 0,6 evento em $20 \mathrm{~min}$ ) e semelhante. Apesar disso, as quedas foram mais frequentes em cordeiros que foram transportados por mais tempo. Não houve lutas durante o transporte.

As variáveis fisiológicas no desembarque e antes do abate estão nos Quadros 4 e 5, respectivamente. A duração

Quadro 2. Duração de cordeiros deitados e número de ruminações, vocalizações e deslocamentos voluntários, em 20 minutos, durante os transportes rodoviários

\begin{tabular}{lcccc}
\hline & \multicolumn{4}{c}{ Transporte } \\
\cline { 2 - 5 } & 1h45min & 3h52min & $7 \mathrm{~h} 30 \mathrm{~min}$ & $10 \mathrm{~h} 30 \mathrm{~min}$ \\
\hline \multicolumn{4}{c}{ Deitar } \\
Percentil 25\% & 0,00 & 0,00 & 0,92 & 0,92 \\
Mediana & $0,00^{\mathrm{b}}$ & $0,00^{\mathrm{b}}$ & $2,45^{\mathrm{a}}$ & $2,87^{\mathrm{a}}$ \\
Percentil 75\% & 0,00 & 0,00 & 5,79 & 6,57 \\
& \multicolumn{5}{c}{ Ruminar } \\
Percentil 25\% & 0,00 & 0,00 & 0,01 & 0,00 \\
Mediana & $0,00^{\mathrm{b}}$ & $0,00^{\mathrm{b}}$ & $0,04^{\mathrm{a}}$ & $0,03^{\mathrm{a}}$ \\
Percentil 75\% & 0,00 & 0,00 & 0,12 & 0,06 \\
& \multicolumn{4}{c}{ Vocalizar } \\
Percentil 25\% & 0,00 & 0,00 & 0,00 & 0,00 \\
Mediana & $0,00^{\mathrm{a}}$ & $0,00^{\mathrm{a}}$ & $0,00^{\mathrm{a}}$ & $0,00^{\mathrm{a}}$ \\
Percentil 75\% & 0,00 & 0,00 & 0,00 & 0,00
\end{tabular}

Letras iguais na mesma linha não diferem estatisticamente pelo teste de Kruskal-Wallis ( $\mathrm{p}>0,05)$. 
Quadro 3. Número de eventos potencialmente traumáticos, em 20 minutos, durante o transporte rodoviário de cordeiros em diferentes durações

\begin{tabular}{|c|c|c|c|c|}
\hline & \multicolumn{3}{|c|}{ Transporte } & \multirow[b]{2}{*}{$10 \mathrm{~h} 30 \mathrm{~min}$} \\
\hline & $1 \mathrm{~h} 45 \mathrm{~min}$ & 3h52min & 7h30min & \\
\hline \multicolumn{5}{|c|}{ Perdas de equilíbrio } \\
\hline Percentil 25\% & 0,00 & 0,00 & 0,00 & 0,00 \\
\hline Mediana & $0,00^{\mathrm{a}}$ & $0,00^{\mathrm{a}}$ & $0,00^{\mathrm{a}}$ & $0,00^{\mathrm{a}}$ \\
\hline Percentil 75\% & 0,14 & 0,00 & 0,04 & 0,00 \\
\hline \multicolumn{5}{|c|}{ Quedas } \\
\hline Percentil 25\% & 0,00 & 0,00 & 0,00 & 0,00 \\
\hline Mediana & $0,00^{\mathrm{a}}$ & $0,00^{\mathrm{a}}$ & $0,00^{\mathrm{ab}}$ & $0,00^{\mathrm{b}}$ \\
\hline Percentil 75\% & 0,00 & 0,00 & 0,00 & 0,03 \\
\hline \multicolumn{5}{|c|}{ Saltar e andar sobre outros animais } \\
\hline Percentil 25\% & 0,00 & 0,00 & 0,00 & 0,03 \\
\hline Mediana & $0,00^{\mathrm{a}}$ & $0,00^{\mathrm{a}}$ & $0,11^{\mathrm{a}}$ & $0,08^{\mathrm{a}}$ \\
\hline Percentil 75\% & 0,19 & 0,07 & 0,34 & 0,24 \\
\hline \multicolumn{5}{|c|}{ Cabeçada } \\
\hline Percentil 25\% & 0,00 & 0,00 & 0,00 & 0,00 \\
\hline Mediana & $0,00^{\mathrm{a}}$ & $0,00^{\mathrm{a}}$ & $0,00^{\mathrm{a}}$ & $0,00^{\mathrm{a}}$ \\
\hline Percentil 75\% & 0,00 & 0,00 & 0,00 & 0,05 \\
\hline \multicolumn{5}{|c|}{ Total de eventos potencialmente traumáticos* } \\
\hline Percentil 25\% & 0,00 & 0,00 & 0,04 & 0,06 \\
\hline Mediana & $0,00^{\mathrm{a}}$ & $0,09^{a}$ & $0,18^{\mathrm{a}}$ & $0,16^{\mathrm{a}}$ \\
\hline Percentil 75\% & 0,67 & 0,34 & 0,35 & 0,28 \\
\hline
\end{tabular}

do transporte não alterou a frequência respiratória e a temperatura corporal dos animais no desembarque e antes do abate. As concentrações de albumina e proteínas totais, no desembarque e antes do abate, foram semelhantes entre os tratamentos.

Os parâmetros neuroendócrinos, adrenalina e cortisol, foram semelhantes entre as diferentes jornadas neste experimento. Também não houve diferenças nas concentrações de glicose, ureia e AGNE no desembarque. Após o descanso pré-abate, as concentrações de glicose estavam linearmente maiores em transportes com maior duração.

No desembarque, as concentrações de CK foram maiores linearmente em cordeiros que foram transportados por mais tempo. Mesmo após o descanso pré-abate, os valores encontravam-se linearmente maiores em transportes mais longos. Os valores de lactato foram semelhantes entre as diferentes jornadas no desembarque e também antes do abate.

As carcaças quentes apresentaram efeito linear decrescente com o aumento da duração das jornadas (Quadro 6); para cada acréscimo de 100 min na jornada, uma carcaça diminuiu seu peso em 0,28 kg. 0 mesmo ocorreu para as carcaças frias, o seu peso diminuiu $0,30 \mathrm{~kg}$ para $100 \mathrm{~min}$ percorridos.

0 rendimento de carcaça quente não foi alterado com o aumento da duração das jornadas, já o rendimento de carcaça fria diminuiu linearmente. Para cada $100 \mathrm{~km}$ percor-

Quadro 4. Variáveis fisiológicas de cordeiros imediatamente após o desembarque em transportes com durações crescentes

\begin{tabular}{|c|c|c|c|c|c|c|c|}
\hline & \multicolumn{4}{|c|}{ Transporte } & \multirow{2}{*}{$\begin{array}{c}\text { Equação de } \\
\text { Regressão }\end{array}$} & \multirow[t]{2}{*}{$\mathrm{R}^{2}$} & \multirow[t]{2}{*}{$\mathrm{CV}(\%)$} \\
\hline & $1 \mathrm{~h} 45 \mathrm{~min}$ & $3 \mathrm{~h} 52 \mathrm{~min}$ & 7h30min & $10 \mathrm{~h} 30 \mathrm{~min}$ & & & \\
\hline FRa (mov./min) & 48,20 & 43,00 & 50,60 & 41,50 & $Y=45,83$ & nsf & 28,55 \\
\hline $\mathrm{TRb}\left({ }^{\circ} \mathrm{C}\right)$ & 39,42 & 39,11 & 39,19 & 39,36 & $Y=39,27$ & ns & 1,20 \\
\hline Albumina (g/dL) & 2,69 & 2,55 & 2,65 & 2,66 & $Y=2,64$ & ns & 9,51 \\
\hline PTc $(g / d L)$ & 6,75 & 6,39 & 7,19 & 7,20 & $Y=6,88$ & ns & 10,24 \\
\hline Adrenalina $(\mathrm{ng} / \mathrm{mL})$ & 1,35 & 1,33 & 1,38 & 1,60 & $Y=1,42$ & ns & 24,01 \\
\hline Glicose (g/dL) & 96,96 & 106,70 & 82,56 & 83,23 & $Y=92,36$ & ns & 14,50 \\
\hline Cortisol (ug/dL) & 20,31 & 18,18 & 12,89 & 29,78 & $Y=20,29$ & ns & 20,10 \\
\hline $\mathrm{CKd}(\mathrm{g} / \mathrm{dL})$ & 236,51 & 181,80 & 388,98 & 447,12 & $\hat{Y}=0,49 X+140,20$ & 0,72 & 25,00 \\
\hline Lactato $(\mathrm{g} / \mathrm{dL})$ & 27,40 & 17,41 & 27,77 & 19,57 & $\mathrm{Y}=23,04$ & ns & 24,00 \\
\hline Ureia (g/dL) & 35,36 & 55,52 & 50,84 & 49,09 & $Y=47,70$ & ns & 29,15 \\
\hline AGNEe $(\mathrm{mmol} / \mathrm{L})$ & 0,07 & 0,08 & 0,03 & 0,04 & $Y=0,06$ & ns & 26,33 \\
\hline
\end{tabular}

$\overline{{ }^{a}}$ Frequência respiratória, ${ }^{\mathrm{b}}$ Temperatura retal, ${ }^{\mathrm{c}}$ Proteínas totais, ${ }^{\mathrm{d}}$ Creatina quinase, ${ }^{\mathrm{e}}$ Ácidos graxos não esterificados, ${ }^{\mathrm{f}}$ não significativo $(\mathrm{p}>0,05)$.

Quadro 5. Variáveis fisiológicas de cordeiros antes do abate, 15 horas após o transporte com durações crescentes

\begin{tabular}{|c|c|c|c|c|c|c|c|}
\hline & \multicolumn{4}{|c|}{ Transporte } & \multirow{2}{*}{$\begin{array}{l}\text { Equação de } \\
\text { Regressão }\end{array}$} & \multirow[t]{2}{*}{$\mathrm{R}^{2}$} & \multirow[t]{2}{*}{$\mathrm{CV}(\%)$} \\
\hline & $1 \mathrm{~h} 45 \mathrm{~min}$ & $3 \mathrm{~h} 52 \mathrm{~min}$ & 7h30min & $10 \mathrm{~h} 30 \mathrm{~min}$ & & & \\
\hline $\mathrm{FRa}(\mathrm{mov} . / \mathrm{min})$ & 36,60 & 34,07 & 34,86 & 35,76 & $\mathrm{Y}=35,32$ & nsf & 26,07 \\
\hline $\operatorname{TRb}\left({ }^{\circ} \mathrm{C}\right)$ & 38,81 & 38,72 & 38,58 & 38,88 & $\mathrm{Y}=38,75$ & ns & 1,37 \\
\hline Albumina (g/dL) & 2,81 & 2,41 & 2,54 & 2,37 & $Y=2,53$ & ns & 10,90 \\
\hline $\operatorname{PTc}(\mathrm{g} / \mathrm{dL})$ & 7,43 & 6,33 & 6,77 & 6,56 & $Y=6,77$ & ns & 10,58 \\
\hline Glicose $(\mathrm{g} / \mathrm{dL})$ & 59,60 & 60,91 & 64,44 & 67,82 & $\hat{Y}=0,016 X+57,59$ & 0,99 & 14,50 \\
\hline Cortisol (ug/dL) & 11,74 & 6,39 & 23,42 & 18,99 & $Y=15,14$ & ns & 21,00 \\
\hline CKd $(\mathrm{g} / \mathrm{dL})$ & 223,55 & 215,76 & 354,92 & 351,21 & $\hat{Y}=0,30 X+179,80$ & 0,70 & 23,01 \\
\hline Lactato $(\mathrm{g} / \mathrm{dL})$ & 12,03 & 16,65 & 12,83 & 12,19 & $Y=13,42$ & ns & 22,40 \\
\hline Ureia $(\mathrm{g} / \mathrm{dL})$ & 59,50 & 64,03 & 53,23 & 50,02 & $Y=56,69$ & ns & 21,80 \\
\hline AGNEe (mmol/L) & 0,33 & 0,43 & 0,32 & 0,27 & $Y=0,40$ & ns & 17,67 \\
\hline
\end{tabular}

$\overline{{ }^{a}}$ Frequência respiratória, ${ }^{\mathrm{b}}$ Temperatura retal, ${ }^{\mathrm{c}}$ Proteínas totais, ${ }^{\mathrm{d}}$ Creatina quinase. ${ }^{\mathrm{e}}$ Ácidos graxos não esterificados, ${ }^{\mathrm{f}}$ não significativo $(\mathrm{p}>0,05)$. 
Quadro 6. Características de carcaça e carne de cordeiros submetidos ao transporte rodoviário em durações crescentes

\begin{tabular}{|c|c|c|c|c|c|c|c|}
\hline & \multicolumn{4}{|c|}{ Transporte } & \multirow{2}{*}{$\begin{array}{l}\text { Equação de } \\
\text { Regressão }\end{array}$} & \multirow[t]{2}{*}{$\mathrm{R}^{2}$} & \multirow[t]{2}{*}{$\mathrm{CV}(\%)$} \\
\hline & $1 \mathrm{~h} 45 \mathrm{~min}$ & $3 \mathrm{~h} 52 \mathrm{~min}$ & 7h30min & $10 \mathrm{~h} 30 \mathrm{~min}$ & & & \\
\hline PCQa (kg) & 17,04 & 16,09 & 15,31 & 15,54 & $\hat{Y}=-0,0028 X+17,00$ & 0,74 & 18,36 \\
\hline $\mathrm{PCFb}(\mathrm{kg})$ & 16,19 & 15,69 & 14,74 & 14,73 & $\hat{Y}=-0,003 X+16,39$ & 0,90 & 18,69 \\
\hline RCQc (\%) & 45,45 & 43,78 & 44,42 & 44,09 & $Y=44,44$ & nsg & 4,99 \\
\hline RCFd (\%) & 44,08 & 43,40 & 42,39 & 42,68 & $\hat{Y}=-0,003 X+43,48$ & 0,77 & 5,02 \\
\hline $\mathrm{pH}$ inicial & 6,77 & - & 6,62 & 6,63 & $Y=6,67$ & ns & 2,62 \\
\hline $\mathrm{pH}$ final & 5,77 & - & 5,63 & 5,65 & $Y=5,68$ & ns & 2,98 \\
\hline CRAe (\%) & 69,01 & 69,59 & 67,30 & 69,33 & $Y=68,81$ & ns & 5,25 \\
\hline $\mathrm{FCf}(\mathrm{kg} / \mathrm{cm} 2)$ & 3,27 & 4,10 & 4,37 & 3,99 & $Y=3,93$ & ns & 35,76 \\
\hline $\mathrm{L}^{*} \mathrm{~g}$ & 32,39 & 34,36 & 35,54 & 34,71 & $Y=34,25$ & ns & 9,92 \\
\hline$a^{*} h$ & 5,76 & 5,90 & 6,17 & 5,94 & $Y=5,94$ & ns & 23,35 \\
\hline$b^{*} \mathrm{i}$ & 8,05 & 7,98 & 7,81 & 8,11 & $Y=7,99$ & ns & 18,04 \\
\hline
\end{tabular}

$\overline{{ }^{a} \text { Peso de carcaça quente, }{ }^{\mathrm{b}}}$ Peso de carcaça fria, ${ }^{\mathrm{c}}$ Rendimento de carcaça quente, ${ }^{\mathrm{d}}$ Rendimento de carcaça fria, ${ }^{\mathrm{e}}$ Capacidade de retenção de água da carne, ${ }^{\mathrm{f}}$ Força de cisalhamento da carne, ${ }^{\mathrm{g}}$ Luminosidade da carne, ${ }^{\mathrm{h}}$ Tendência para o vermelho da carne, ${ }^{\mathrm{i}}$ Tendência para amarelo da carne, ${ }^{\mathrm{g}}$ não significativo $(\mathrm{p}>0,05)$. Valores de $\mathrm{pH}$ inicial e final do transporte $3 \mathrm{~h} 52 \mathrm{~min}$ foram perdidos.

ridos o rendimento de carcaça fria diminuiu 0,3 ponto percentual, portanto, houve perdas também dos constituintes não-carcaça.

Os parâmetros envolvidos com a qualidade da carne dos cordeiros também estão no Quadro $6.0 \mathrm{pH}$ inicial das carcaças foi semelhante entre os tratamentos, seu valor médio foi de $6,67 \pm 0,03$. 0 mesmo ocorreu quando se avaliou o $\mathrm{pH}$ após $24 \mathrm{~h}, 5,68 \pm 0,02$ foi a média dos valores. A capacidade de retenção de água também foi semelhante entre os tratamentos. A coloração, medida pelos parâmetros $\mathrm{L}^{*}, \mathrm{a}^{*} \mathrm{e} \mathrm{b*} \mathrm{e}$ a força de cisalhamento não diferiram com o aumento da duração dos transportes.

\section{DISCUSSÃO}

A semelhança deste experimento, Knowles et al. (1998) mostraram que os cordeiros deitaram, com maior frequência, a partir de 6h. Para Brule et al. (2001), a ruminação indica que o animal está adaptado a um ambiente não familiar. Das et al. (2001), avaliando o transporte de caprinos, também relataram ruminações com durações curtas. A ruminação durante o transporte é frequentemente citada como baixa (Kenny \& Tarrant 1987, Das et al. 2001). Nota-se que, neste experimento, a frequência da ruminação deu-se com o aumento da duração de animais deitados. Portanto, parece haver uma adaptação crescente com o aumento da duração das jornadas. Esta hipótese não é confirmada quando se avalia os demais parâmetros.

Para Forkman et al. (2007), orelhas armadas e pescoço em posição vertical são indícios importantes de medo. Já a vocalização não é um bom sinal de medo para ovinos (Grandin 2010), o que pode explicar a frequência dessa variável neste experimento.

Cockram et al. (2010), corroborando com este ensaio, mostraram que a soma dos eventos potencialmente traumáticos foi menor do que 5 eventos hora-1. A condução do veículo, sempre em prol da minimização dos efeitos maléficos do transporte, pode explicar os resultados destas variáveis nesta pesquisa.

As frequências respiratórias dos animais deste experimento, no desembarque e antes do abate, não são conside- radas valores de ovinos estressados por calor, partindo dos relatos de Hales \& Brown (1974). Também, de acordo com os valores de temperatura corporal de ovinos apresentados por Cunningham $(2004)\left(38,5\right.$ a $\left.39,9^{\circ} \mathrm{C}\right)$, em quaisquer situações deste ensaio, os valores encontram-se dentro da normalidade.

As concentrações de albumina e proteínas totais, no desembarque e antes do abate, encontram-se dentro dos valores normais citados por Kaneko et al. (2008). 0 aumento de suas concentrações pode indicar desidratação (Al-Haidary et al. 2012). Portanto, pode-se afirmar que não houve desidratação para quaisquer tratamentos.

Adrenalina e cortisol são constantemente relacionados ao metabolismo de adaptação ao estresse e regulação do metabolismo basal (Ferguson \& Warner 2008). A semelhança entre os tratamentos é coerente com os achados de glicose, ureia e AGNE no desembarque, já que nenhuma dessas variáveis foi diferente com a duração crescente dos transportes.

No desembarque, os valores de glicose, para quaisquer áreas transportes, encontram-se levemente acima dos valores referenciados por Kaneko et al. (2008) como normais (50-80 $\left.\mathrm{mg} \mathrm{dL}^{-1}\right)$. Concentrações de glicose acima dos valores normais foram encontradas em ovinos após transporte; e aliam o fato ao aumento do estresse (Ekiz et al. 2012). Após o descanso pré-abate, as concentrações de glicose estavam normais para todos os transportes, apesar de os animais que foram transportados em uma maior duração apresentarem maior concentração de glicose. Talvez o descanso pré-abate necessite ser diferente de acordo com a duração do transporte, contudo, ao contrário de pesquisas com suínos e frangos (Dalla Costa et al. 2008, Rui et al. 2011), não foi encontrado artigos com ovinos que justifiquem esta ação neste contexto específico.

As concentrações de lactato e CK são utilizadas para averiguação do aumento do esforço físico e de chances de contusões, respectivamente (Broom \& Fraser, 2010). Portanto, o aumento da duração do transporte resultou em maiores possibilidades de lesões teciduais. Contrariamente, os valores de lactato não indicaram fadiga. 
Diferente deste trabalho, Tarrant (1990) citou que as perdas nas carcaças são mais susceptíveis a não ruminantes e que em bovinos são insignificantes até 24 horas de jejum. Kadim et al. (2006) também não encontraram perdas de carcaças quente e fria de caprinos que foram transportados por $100 \mathrm{~km}$ durante 2 horas. Nota-se também neste ensaio que as perdas nas carcaças dos animais transportados por mais tempo foram mais acentuadas do que as dos constituintes não-carcaça, já que o rendimento de carcaça fria diminuiu. Uma hipótese para a elevada perda em peso nas carcaças é que, o escore de gordura neste experimento foi mediano. Os escores de gordura das carcaças, para todos os tratamentos, foram semelhantes $(p \geq 0,05)$, sua média foi de $2,53 \pm 0,05$ pontos, em uma escala de 1 a 5 . Thompson et al. (1987) relataram que em ovinos que possuíam carcaças com maior espessura de gordura, e foram submetidos ao jejum de sólidos por 96 horas antes do abate, perderam $6 \%$ de peso da carcaça em relação a animais que não passaram por jejum; em ovinos que possuíam carcaças com menor espessura de gordura, ainda no trabalho de Thompson et al. (1987), esta perda foi de $12 \%$.

Partindo do resultado das perdas em peso das carcaças deste experimento, criadores de ovinos devem buscar empresas mais próximas de sua propriedade para abates dos cordeiros, pois a duração dos transportes interfere fortemente no peso da carcaça. Simulações de perdas quantitativas das carcaças devem fazer parte de planilhas de custos para tomadas de decisões mais adequadas.

Os pHs inicial e final deste ensaio estão dentro do considerado normal por Lawrie (2005), o que manteve constante e normal a capacidade de retenção de água (Huff-Lonergan \& Lonergan 2005). Os valores da força de cisalhamento da carne, em todos os grupos, estão abaixo de 11 kgf, considerados por Souza et al. (2004) como limite superior para considerar a carne como macia. Contrariamente a este experimento, Carter \& Gallo (2008) relataram que o transporte de ovinos para o abatedouro frigorífico resultou em depleção do glicogênio e consequente $\mathrm{pH}$ final mais alto.

Variações de 30,03 a 49,47 para luminosidade ( $\mathrm{L}^{*}$ ), de 8,24 a 23,53 para intensidade de vermelho $\left(a^{*}\right)$ e de 3,38 a 11,10 para intensidade de amarelo ( $b^{*}$ ) são citadas em carnes de ovinos (somente tecido muscular) (Sañudo et al. 2000). Nota-se que todos os tratamentos apresentaram, quando comparados com a referência acima, carnes com mais brilho ( $\left.\mathrm{L}^{*}\right)$ e intensidade de amarelo ( $\left.\mathrm{b}^{*}\right)$. A intensidade de vermelho $\left(\mathrm{a}^{*}\right)$ está abaixo, para quaisquer transportes, da referência, talvez resultado da diferença do genótipo e diferenças no sistema de criação e características intrínsecas dos alimentos.

\section{CONCLUSÕES}

Os parâmetros comportamentais e fisiológicos não evidenciam a diminuição do bem-estar dos cordeiros para quaisquer durações do transporte.

A chance de lesões teciduais é maior em transportes mais longos (intervalo de $1 \mathrm{~h} 45 \mathrm{~min}$ a $10 \mathrm{~h} 30 \mathrm{~min}$ ).

Os pesos das carcaças quente e fria e o rendimento da carcaça fria foram menores em transportes mais longos. Partindo da premissa que a diminuição destes valores é re- sultado da diminuição do bem-estar, os cordeiros transportados em uma maior duração passam por condições mais adversas.

Os indicativos de qualidade da carne não apresentaram alterações significativas para quaisquer durações do transporte (intervalo de $1 \mathrm{~h} 45 \mathrm{~min}$ a $10 \mathrm{~h} 30 \mathrm{~min}$ ).

Agradecimentos.- Ao Conselho Nacional de Desenvolvimento Tecnológico (CNPq) por financiar este projeto. Ao CNPq, à Coordenação de Aperfeiçoamento de Pessoal de Nível Superior (CAPES) e à Fundação de Amparo à Pesquisa do Estado de Minas Gerais (FAPEMIG) por concederem bolsas de estudo ou pesquisa aos autores. À FAPEMIG por financiar a publicação deste artigo.

\section{REFERÊNCIAS}

Al-Haidary A.A., Aljumaah R.S., Alshaikh M.A., Abdoun K.A., Samara E.M., Okab A.B. \& Alfuraiji M.M. 2012. Thermoregulatory and Physiological Responses of Najdi Sheep Exposed to Environmental Heat Load Prevailing in Saudi Arabia. Pak. Vet. J. 32:515-519.

Brasil 1997. Regulamento da inspeção industrial e sanitária de produtos de origem animal: aprovado pelo Decreto no 30.691, de 29-03-52, alterado pelos Decretos nํㅗ 1.255 de 25-06-62, 1.236 de 02-09-94, no 1.812 de 08-02-96 e no 2.244 de 04-06-97. Departamento de Inspeção de Produtos de Origem Animal, Divisão de Normas Técnicas, Ministério da Agricultura e do Abastecimento.

Broom D.M. \& Fraser A.F. 2010. Comportamento e Bem-Estar dos Animais Domésticos. 4ª ed. Manole, São Paulo. 438p.

Brule A., Chupin J.M., Capdeville J., Lucbert J. \& Sangnac C. 2001. Le transport routier des bovins: effets des conditions de transport surlebiên-être des animaux et sur les pertes techniques. Renc. Rech. Rum. 8:119-128.

Carter L.M. \& Gallo C.B. 2008. Efectos del transporte prolongado por vía terrestre y cruce marítimo en transbordador sobre pérdidas de peso vivo y características de la canal en cordeiros. Arch. Med. Vet. 40:259-266.

Cockram M.S., Kent J.E., Goddard P.J., Waran N.K., Micgilp I.M., Jackson R.E., Muwanga G.M. \& Prytherch S. 2010. Effect of space allowance during transport on the behavioural and physiological responses of lambs during and after transport. Anim. Sci. 62(3):461-477.

Cunningham J.G. 2004. Tratado de Fisiologia Veterinária. 3ª ed. Guanabara Koogan, Rio de Janeiro. 596p.

Dalla Costa O.A., Costa M.J.R.P.D., Ludke J.V., Coldebella A., Kich J.D., Peloso J.V. \& Dalla Roza D. 2008. Tempo de jejum dos suínos no manejo pré-abate sobre a perda de peso corporal, o peso do conteúdo estomacal e a incidência de úlcera esofágica-gástrica. Ciência Rural 38:199-205.

Das K.S., Srivastava B.B. \& Das N. 2001. Standing orientation and behaviour of goats during short-haul road transportation. Technical Note, Small Rum. Res. 41:91-94.

Ekiz B., Ekiz E.E., Kocak O., Yalcintan H. \& Yilmaz A. 2012. Effect of pre-slaughter management regarding transportation and time in lairage on certain stress parameters, carcass and meat quality characteristics in Kivircik lambs. Meat Sci. 90(4):967-976.

Ferguson D.M. \& Warner R.D. 2008. Have we underestimated the impact of pre-slaughter stress on meat quality in ruminants? Review. Meat Sci. 80:12-19.

Fisher A.D., Niemeyer D.O., Lea J.M., Lee C., Paull D.R., Reed M.T. \& Ferguson D.M. 2010. The effects of 12,30 , or 48 hours of road transport on the physiological and behavioural responses of sheep. J. Anim. Sci. 88:2144-2152.

Forkman B., Boissy A., Meunier-Salaün M.C., Calani E. \& Jones R.B. 2007. Critical review of fear tests used on cattle, pigs, sheep, poultry and horses. Physiol. Behav. 92:340-397.

Gomide L.A.M., Ramos E.M. \& Fontes P.R. 2006. Tecnologia de abate e tipificação de carcaças. UFV, Viçosa.

Grandin T. 2010. Auditing animal welfare at slaughter plants: a review. Meat Sci. 86:56-65.

Hales J.R.S. \& Brown G.D. 1974. Net energetic and ther- moregulatory ef- 
ficiency during panting in the sheep. Comp. Biochem. Physiol. 49:413422.

Huff-Lonergan E. \& Lonergan S.M. 2005. Mechanisms of water-holding capacity of meat: the role of postmortem biochemical and structural changes. Meat Sci. 71(1):194-204.

Kadim I.T., Mahgoup O., Al-Marzooqi W., Khalaf S., Al-Sinawi S.S.H. \& Al-Amri I.S. 2006. Effects of transportation at high ambient temperatures on physiological responses, carcass and meat quality characteristics of three breeds of Omani goats. Meat Sci. 73(4):626-634.

Kaneko J.J., Harvey J.W. \& Bruss M.L. 2008. Clinical Biochemistry of Domestic Animals. 6th ed. Academic Press, New York. 916p.

Kenny F.J. \& Tarrant P.V. 1987. The physiological and behavioural responses of crossbred Friesian steers to short-haul transport by road. Livest. Prod. Sci. 17:63-75.

Knowles T.G., Warriss P.D., Brown S.N. \& Edwards J.E. 1998. Effects of stocking density on lambs being transported by road. Vet. Rec. 142(19):503-509.

Lawrie R.A. 2005. Ciência da Carne [Meat Sci.]. $6^{\underline{a}}$ ed. Artmed, Porto Alegre.

Leme T.M.C., Titto E.A.L., Titto C.G., Amadeu C.C.B., Neto P.F., Vilela R.A. \& Pereira A.M.F. 2012. Influence of transportation methods and pre-slaughter rest periods on cortisol level in lambs. Small Ruminant Res. 107 (1):8-11.

Moberg G.P. \& Mench J.A. 2000. The Biology of Animal Stress: basic principles and implications for animal welfare. CABI Publishing. 377p.

Nakamura M. \& Katoh K. 1985. Influence of thawing method on several properties of rabbit meat. Bully. Ishik. Prefect. 11(1):45-49.

National Research Council 2007. Nutrient Requirements of Small Ruminants: Sheep, Goats, Cervids and New World Camelids. 7th ed. National Academy Press, Washington, D.C. 362p.
Paranhos da Costa M.J.R., Quintiliano M.H. \& Tseimazides S.P. 2013. Boas práticas de manejo: transporte. Ministério da Agricultura, Pecuária e Abastecimento, Brasília. 56p.

Petherick J.C. \& Phillips C.J.C. 2009. Space allowances for confined livestock and their determination form allometric principles. Appl. Anim. Behav. Sci. 117:1-12.

Rui B.R., Angrimani D.D.S.R. \& Silva M.A.A.D. 2011. Pontos críticos no manejo pré-abate de frango de corte: jejum, captura, carregamento, transporte e tempo de espera no abatedouro. Ciência Rural 41(7):1290-1296.

Sañudo C., Campo M.M., Sierra I., María G.A., Olleta J.L. \& Santolaria P. 1997. Breed effect on carcass and meat quality of suckling lambs. Meat Sci. 46(4):357-365.

Sañudo C., Enser M.E., Campo M.M., Nute G.R., María G., Sierra I.E. \& Wood J.D. 2000. Fatty acid composition and sensory characteristics of lamb carcasses from Britain and Spain. Meat Sci. 54(4):339-346.

Souza X.R., Bressan M.C., Pérez J.R.O., Faria P.B., Vieira J.O. \& Kabeya D.M. 2004. Efeitos do grupo genético, sexo e peso ao abate sobre as propriedades físico-químicas da carne de cordeiros em crescimento. Ciênc. Tecnol. Aliment. 24(4):543-549.

Tadich N., Gallo C., Brito M.L. \& Broom D.M. 2009. Effects of weaning and $48 \mathrm{~h}$ transport by road and ferry on some blood indicators of welfare in lambs. Livest Sci. 121(1):132-136. Tarrant P.V. 1990. Transport of cattle by road. Appl. Anim. Behav. Sci. 28:153-170.

Thompson J.M., O’Halloran W.J., McNeil D.M.J., Jackson-Hope N.J. \& May T.J. 1987. The effect of fasting on liveweight and carcass characteristics in lambs. Meat Sci. 20:293-309.

Wheeler T.L., Shackelford S.D. \& Koohmaraie M. 2005. Shear force procedures for meat tenderness measurement. Clay Center, N.E. Roman \& L. Hruska U.S. Meat Animal Research Center. 7p. Disponível em <http:// www.ars.usda.gov/SP2UserFiles/Place/54380530/protocols/ShearForceProcedures.pdf> Acesso em: 22 fev. 2012. 